\title{
Prognostic value of increase in transcript levels of Tp73 $\Delta$ Ex2-3 isoforms in low-grade glioma patients
}

\author{
M Wager,', J Guilhot ${ }^{2}$, J-L Blanc', S Ferrand', S Milin ${ }^{3}$, B Bataille', F Lapierre', S Denis ${ }^{4}$, T Chantereau ${ }^{4}$, \\ C-J Larsen ${ }^{4}$ and L Karayan-Tapon ${ }^{4}$ \\ 'Neurosurgery Department, University Hospital, Poitiers, France; ${ }^{2}$ Clinical Research Centre, University Hospital, Poitiers, France; ${ }^{3}$ Department of \\ Pathology, University Hospital, Poitiers, France; ${ }^{4}$ Laboratory of Molecular Oncology, University Hospital, Poitiers, France
}

\begin{abstract}
Glial tumours are a devastating, poorly understood condition carrying a gloomy prognosis for which clinicians sorely lack reliable predictive parameters facilitating a sound treatment strategy. Tp73, a p53 family member, expresses two main classes of isoforms transactivatory activity (TA)p73 and $\Delta \mathrm{TAp} 73$ - exhibiting tumour suppressor gene and oncogene properties, respectively. The authors examined their expression status in high- and low-grade adult gliomas. Isoform-specific real-time reverse transcriptionpolymerase chain reaction was used for the analysis of Tp73 isoform transcript expression in a series of $5 \mathrm{I}$ adult patients harbouring glial tumours, in order to compare tumour grades with each other, and with non-tumoural samples obtained from epileptic patients as well. Our data demonstrate increase of TAp73 and $\Delta T A p 73$ transcript levels at onset and early stage of the disease. We also show that $\Delta \mathrm{E} \times 2-3$ isoform expression in low-grade tumours anticipates clinical and imaging progression to higher grades, and correlates to the patients' survival. Expression levels of PI promoter generated Tp73 isoforms - and particularly $\Delta$ Ex2 -3 - indeed allow for prediction of the clinical progression of low-grade gliomas in adults. Our data are the first such molecular biology report regarding low-grade tumours and as such should be of help for sound decision-making
\end{abstract}

British Journal of Cancer (2006) 95, 1062-1069. doi:I0.1038/sj.bjc.66034 I0 www.bjcancer.com

(c) 2006 Cancer Research UK

Keywords: Tp73 isoforms; low-grade gliomas; prognosis; quantitative reverse-transcription polymerase chain reaction (QRT-PCR)

Glioblastomas are divided into primary or de novo, and secondary tumours originating from low-grade (World Health Organization WHO - grade II) progressing to high-grades, that is anaplastic (WHO grade III) gliomas and eventually to highly malignant glioblastomas (WHO grade IV). Although our knowledge of the molecular mechanisms involved in tumorigenesis and progression has significantly improved over fairly recent years, this has yet to result in an improved therapeutic strategy. To date, no reliable parameter has allowed for anticipation of clinical progression from lower to higher grades. Such criteria would be of paramount importance in therapeutic management of these patients.

The Tp73 gene, a close relative of the tumour suppressor Tp53 gene, contains two independent promoters P1 and P2 (Melino et al, 2002). $\mathrm{P} 1$ promoter controls the TAp73 transcripts containing exons $1-3$ that encode $\mathrm{N}$-terminal sequences with transactivatory activity (TA), and $\Delta \mathrm{Ex} 2, \Delta \mathrm{Ex} 2-3$ and $\Delta \mathrm{N}^{\prime}$ transcripts collectively designate $\Delta$ TAp73 that lack a fully competent TA domain (Stiewe et al, 2002a,b). The TAp73 isoform is capable of inducing cell cycle growth arrest and apoptosis and is also involved in the response of $\mathrm{p} 53$ to death stimuli (Flores et al, 2002). These features are consistent with a tumour suppressor function. In contrast, the $\Delta \mathrm{TA}$ transcripts do not induce cell growth arrest or

\footnotetext{
*Correspondence: Dr M Wager, Service de Neurochirurgie, H3A Tour Jean Bernard, 350 Avenue Jacques Cœur, CHU La Miletrie, Poitiers Cedex 8602I, France; E-mail: m.wager@chu-poitiers.fr

Received 24 March 2006; revised 21 August 2006; accepted 21 August 2006
}

cell death. Significantly, their overexpression in NIH3T3 cells induces malignant transformation and tumour growth in nude mice (Stiewe et al, 2002a, b), which is consistent with an oncogene status. The P2 promoter located downstream of exon $3^{\prime}$ controls $\Delta$ Np73 transcripts lacking the TA domain that likewise exhibit antiapoptotic activity and are therefore functionally similar to $\Delta$ TAp73 transcripts (Grob et al, 2001; Nakagawa et al, 2002). $\triangle \mathrm{Np73}$ has been shown to facilitate the immortalisation of MEFs and to cooperate with oncogenic Ras in transforming primary fibroblasts in vitro and in inducing MEF-derived fibrosarcomas in vivo in nude mice (Petrenko et al, 2003). Because the tetramerisation domain and the DNA-binding domain of the TAp73 protein are conserved in $\Delta \mathrm{Np} 73$ and $\Delta \mathrm{TAp} 73$ isoforms, both have been shown to exert a dominant-negative activity on TAp73 and p53 through oligomerisation with the two proteins and/or by competition for p53/TAp73 target genes (Stiewe et al, 2002a,b). Consistent with these data, upregulation of the $\Delta \mathrm{Np} 73$ isoform has been described in neuroblastomas with poor prognosis and thereby defines this parameter as a strong and independent predictor of such poor prognosis (Casciano et al, 2002). Moreover Bergamaschi et al (2003) and Irwin et al (2003) have shown that TAp73 is induced by a wide variety of chemotherapeutic agents and that chemosensitivity is related to TAp73 function.

In view of the potential relevance of $\mathrm{Tp} 73$ to tumorigenesis, the expression status of the gene has been addressed in various tumour types. Significant results have been achieved only in more recent works taking into account the recently described $\triangle \mathrm{TAp} 73$ transcripts by using appropriate couples of primers, designed to specifically assess contribution of the $\Delta \mathrm{TAp} 73$ isoforms among the 
different P1-generated transcripts collectively referred to as P1 transcripts ( $\mathrm{Ng}$ et al, 2000; Cui et al, 2005). The most convincing evidence to date comes from a work on a series of 100 ovarian tumours of all the known histological types (Concin et al, 2004). On the whole, these data point to the activation of the $\mathrm{P} 1$ promoter and the deregulation of $\mathrm{P} 1$-controlled $\Delta \mathrm{TA}$ p73 isoforms in the tumorigenic process. Furthermore, because the $\Delta \mathrm{N}^{\prime} \mathrm{p} 73$ isoform is upregulated in ovarian tumours but not in other cancers, the data argue in favour of a selective deregulation among the P1 transcripts in accordance with types of cancer.

We have undertaken a prospective study in order to determine the involvement of Tp73 isoforms in gliomagenesis and their prognostic relevance. In the first part we performed a global analysis of P1- and P2- generated transcripts on high- and lowgrade gliomas, whereas in the second part we focused on low-grade gliomas and performed a discriminant analysis of P1- generated transcripts.

\section{PATIENTS AND METHODS}

\section{Patients}

Tissues from 51 adult patients harbouring glial tumours were successively collected during surgery at the Department of Neurological Surgery, University of Poitiers, France, with signed informed consent of all patients and the approval of the ethics committee of the Poitou-Charentes area. These patients were free from any past medical history, especially with regards to brain surgery, brain radiation therapy or chemotherapy. Our series also included three tumour-free patients, operated for refractory epilepsy, obtained from Neurobiotec ${ }^{\circledR}$ (Lyon, France) in compliance with the ethics committee. For each patient a sample was collected at the presumed higher-grade region and named the 'central' sample. Whenever deemed reasonable - that is without significant additional functional risk - a second sample was harvested in the vicinity of the tumour and called 'peripheral'.

All patients were treated between January 2002 and May 2003. Distribution of patients in groups according to the WHO pathology classification appears in Table 1. Twenty-nine patients underwent tumour resection, whereas 22 benefited from stereotactic biopsy. The first series, dedicated to Tp73 involvement, included 28 high-grade and 11 low-grade patients (Table 1A), and 12 were added to this group in a second series focusing on Tp73 isoforms (Table 1B). All low-grade patients had non-enhancing tumours on MRI imaging, and pathology-proven low-grade tumours. In all cases progression to a higher grade was pathology-proven. Tumour diagnosis and grading were established according to the WHO criteria (Kleihues and Cavenee, 2000) and were systematically revised by two expert neuropathologists.

\section{Analysis of Tp73 isoform transcripts}

Tissue harvesting and preparation Per operative pathology exam allowed to check glial tumour diagnosis with samples obtained either from open-sky surgery or stereotactic biopsies. Each tumour

Table I (A) Tp73 global analysis aimed to show Tp73 involvement in this first series of patients. (B) Tp73 isoforms discrimination aimed to show specific isoform involvement on this second series of low-grade tumours patients only. (C) Normalised expression levels of Tp73 isoforms in non-tumoural (epileptic) and in low- and high-grade gliomas

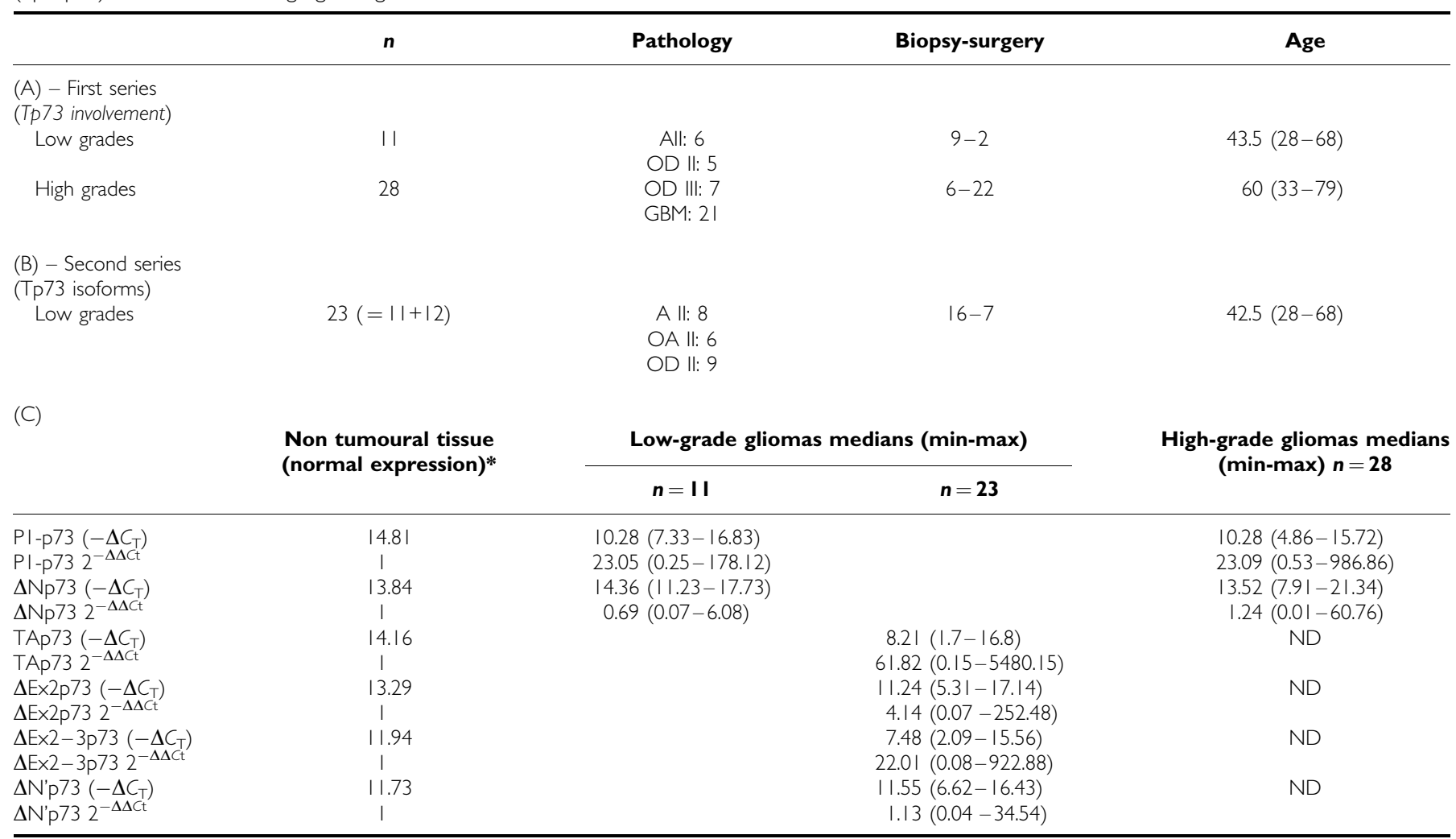

$\mathrm{A}=$ fibrillary astrocytoma; $\mathrm{OA}=$ oligoastrocytoma; $\mathrm{OD}=$ oligodendroglioma; $\mathrm{GBM}=$ glioblastoma. II, III: tumour grades according to the World Health Organization. Results are expressed in $\Delta C_{\mathrm{t}}$ which is the threshold cycle differences (CtGAPDH-CtGene) and in $2^{-\Delta \Delta C t}$ which is the comparative threshold between non-tumoural and tumoural tissue where $\Delta \Delta C_{\mathrm{t}}=\Delta C_{\mathrm{t} \text { Tumoural }}-\Delta C_{\mathrm{t} \text { NonTumoural }}$ and represents fold change value between tumoural and non-tumoural tissue. $\mathrm{Pl}-\mathrm{p} 73, \mathrm{TAp} 73, \Delta \mathrm{E} \times 2 \mathrm{p} 73$ and $\Delta \mathrm{E} \times 2-3 \mathrm{p} 73$ show significantly increased transcript levels as compared to non-tumoural epileptic tissues $(P<10-3, P<10-4, P=0.01$ and $P=0.03$, respectively). There is no difference of expression between low- and high-grade gliomas. (*) Non-tumoural tissue value was based on the analysis of three epileptic patients. The mean of $\Delta C_{\mathrm{t}}$ values was considered as normal expression. (ND: not done). 
sample was divided into two parts: one was dedicated to smears, the second was immediately frozen in liquid nitrogen in the operating room, and stored at $-80^{\circ} \mathrm{C}$ until usage.

RNA isolation and complementary DNA preparation Total RNA was extracted from tumour tissues using the RNAeasy ${ }^{\circledR}$ Mini Kit (Qiagen, Courtaboeuf, Paris, France) according to the manufacturer's instructions with minor modifications, for exclusion of contaminated genomic DNA. The spin-column membranes were treated with DNase (Qiagen) for $15 \mathrm{~min}$ at room temperature before elution. Ten microliters of DNAse-treated total RNA was transcribed into cDNA using Superscript ${ }^{\mathrm{TM}}$ II RnaseH - and random hexamers (Invitrogene ${ }^{\circledR}$, Courtaboeuf, Paris, France).
Real-time reverse transcription-polymerase chain reaction and relative quantification We assessed levels of $\mathrm{Tp} 73$ isoform mRNA transcripts by real-time quantitative $P C R$ in the $A B I$ PRISM 7000 sequence detection system (Applera, Courtaboeuf, Paris, France). Primer sequences are listed in Figure 1. All primers were queried against the no redundant Human Genome Database. Probe sequence is $5^{\prime}$-CAGTTCAATCTGCTGAGCA- $3^{\prime}$. All primer pairs detected a unique specific cDNA. The forward primers for $\Delta \mathrm{Ex} 2 \mathrm{p} 73$ as well as for $\Delta \mathrm{Ex} 2-3 \mathrm{p} 73$ were designed to specifically recognise on the exon-exon boundaries (exon1/exon3 for $\Delta \mathrm{Ex} 2 \mathrm{p} 73$ and exon1/exon 4 for $\Delta \mathrm{Ex} 2-3 \mathrm{p} 73$ ). Indeed, these primers could not hybridise on TAp73 transcripts as the selected boundaries exist only in the variants forms. The forward primer

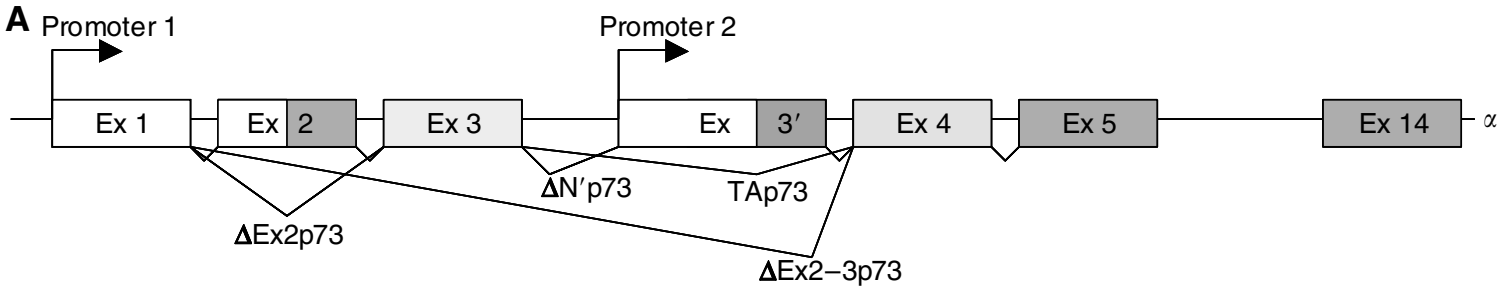

B

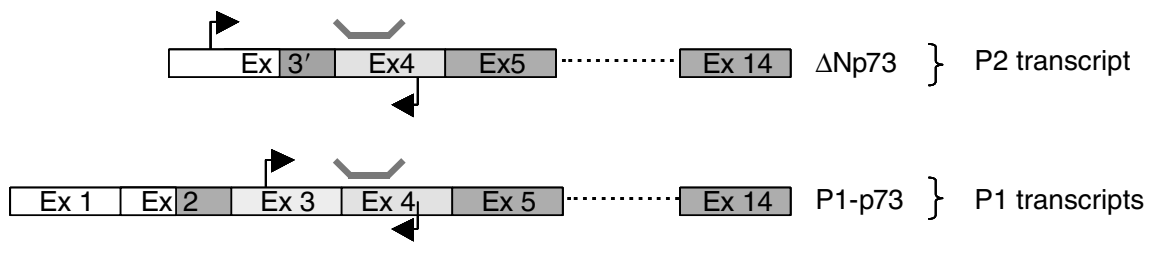

C

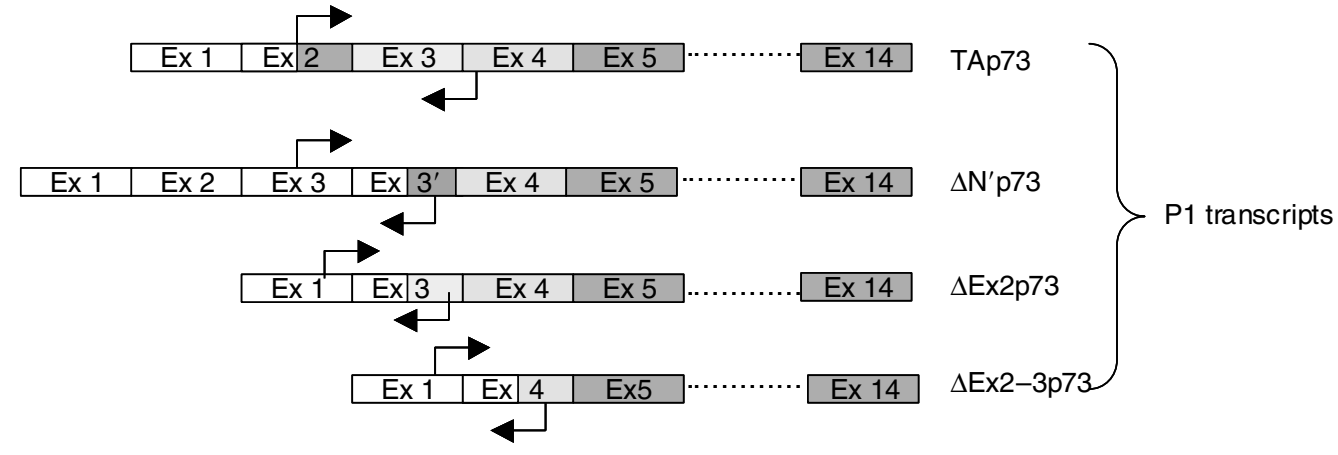

D

\begin{tabular}{|l|c|c|}
\hline & Sense $\left(5^{\prime}-3^{\prime}\right)$ & Antisense $\left(5^{\prime}-3^{\prime}\right)$ \\
\hline P1-p73 & GGAGGGCATGACTACATCTGTCA & GCGCGGCTGCTCATCT \\
\hline$\Delta$ Np73 & GCGCCTACCATGCTGTACGT & GCGCGGCTGCTCATCT \\
\hline TAp73 & GCACCACGTTTGAGCACCTC & GCAGATTGAACTGGGCCATG \\
\hline$\Delta$ Ex2p73 & ACGGCTGCAGGGAACCA & TGCCCTCCAGGTGGAAGAC \\
\hline$\Delta$ Ex2-3p73 & TGCAGGCCCAGTTCAATCTG & CGGTGTTGGAGGGGATGAC \\
\hline$\Delta$ N'p73 $^{\prime} p$ GGATTCCAGCATGGACGTCTT & AGAGGCTCCGCAGCTAGTGA \\
\hline
\end{tabular}

Figure I (A) Genomic organisation of the NH2 terminus of Tp73. Arrows indicate transcriptional start sites. Exons are represented as boxes. The colours indicate the number of the exons, and white untranslated sequences. Exons 2 and 3 encode for the transactivation domain of the full-length p73 protein. Spliced transcripts regulated by Promoter I are labelled TAp3, $\Delta \mathrm{E} \times 2 \mathrm{p} 73, \Delta \mathrm{E} \times 2-3 \mathrm{p} 73$ and $\Delta \mathrm{N}$ 'p 73 . (B) Localisation of the primer pairs (arrows) and probe (red line) used in the global analysis of promoter I encoded transcripts (PI-p73) and promoter 2 encoded transcript ( $\Delta$ Np73) by real-time PCR assays. (C) Localisation of the primer pairs (arrows) used for isoform-specific real-time PCR assays. (D) Sequences of the sense- and antisense-primers used for the global analysis of promoter I generated transcripts (PI-p73) and promoter 2 generated transcript $(\Delta N p 73)$ and those of isoform-specific amplification of individual $\mathrm{NH} 2$ terminus Tp73 isoforms. The forward primers for $\Delta \mathrm{E} \times 2 \mathrm{p} 73$ as well as for $\Delta \mathrm{E} \times 2-3 \mathrm{p} 73$ were designed on the exon-exon boundary (exonl/exon3 for $\Delta \mathrm{Ex} 2 \mathrm{p} 73$ and exon l/exon 4 for $\Delta \mathrm{E} \times 2-3 \mathrm{p} 73$ ). These primers could not hybridise on TAp 73 transcripts as the boundary exists only in the variants forms. The forward primer used for specific TAp73 amplification is localised in exon 2 and the reverse on the exon3/exon 4 boundary and could only amplifiy TAp73. Specificity for $\Delta \mathrm{N}^{\prime} \mathrm{p} 73$ was achieved by the unique combination of the upstream (on exon3) and downstream (on exon $3^{\prime}$ ) primers. 
used for specific TAp73 amplification is localised in exon 2 and the reverse on the exon3/exon 4 boundary and could only amplify TAp73. Specificity for $\Delta N^{\prime} p 73$ was achieved by the unique combination of the upstream (on exon3) and downstream (on exon $3^{\prime}$ ) primers. However, the specific amplification of TAp73, $\Delta \mathrm{Ex} 2 \mathrm{p} 73, \Delta \mathrm{Ex} 2-3 \mathrm{p} 73$ and $\Delta \mathrm{N}^{\prime} \mathrm{p} 73$ transcripts was checked on gel and gives as expected a single band of $168,115,178$ and $90 \mathrm{pb}$. This specific amplification was confirmed by sequencing the products.

The reactions were carried out first by using the Taqman ${ }^{\circledR}$ chemistry for the global screening of P1-generated p73 transcripts $(\mathrm{P} 1-\mathrm{p} 73)$ and P2-generated transcripts $(\Delta \mathrm{Np} 73)$. We then used SyberGreen chemistry assessing of discriminant Tp73 isoform expression. Briefly, the PCR reactions were performed in $25 \mu \mathrm{l}$ reaction volumes consisting of $1 \times$ Taqman Universal PCR Master-Mix or SyberGreen Universal PCR Master-Mix (Applera), one out of 20 of the reverse transcription reaction, $300 \mathrm{~nm}$ of each primer and $200 \mathrm{~nm}$ of probe when Taqman chemistry was used. The samples were submitted to amplification as follows: heating at $50^{\circ} \mathrm{C}$ for $2 \mathrm{~min}, 95^{\circ} \mathrm{C}$ for $10 \mathrm{~min}$ followed by 40 cycles at $95^{\circ} \mathrm{C} \times 15 \mathrm{~s}, 60^{\circ} \mathrm{C} \times 1 \mathrm{~min}$. Each RNA sample was tested in duplicate and a negative control was included in every plate. The computed tomography value was defined as the cycle number $\left(C_{\mathrm{t}}\right)$ at which the fluorescence crossed the threshold. Range of threshold cycles was $C_{\mathrm{t}}: 25-37.6$ for P1-TAp73, $C_{\mathrm{t}}: 26.9-39.8$ for $\Delta \mathrm{Np} 73 \quad C_{\mathrm{t}}: 22.89-39.43$ for TAp73, $C_{\mathrm{t}}: 26.73-37.33$ for $\Delta \mathrm{Ex} 2 \mathrm{p} 73, C_{\mathrm{t}}: 23.24-38.75$ for $\Delta \mathrm{Ex} 2-3 \mathrm{p} 73$ and $C_{\mathrm{t}}: 30.32-36.36$ for $\Delta N^{\prime} p 73$. Results were normalised using the endogenous reference GAPDH. The threshold cycle differences is given in Table 1. The amplification efficiency of the reference gene was similar to that of the target genes. We employed the relative quantification method described in Applied Biosystems User Bulletin No. 2 (Applied Biosystem User Bulletin, 1997) and by Livak and Schmittgen (2001), in which the amount of target, normalised to the endogenous reference GAPDH and relative to the non-tumoural epileptic tissue, is indicated by the $2^{-\Delta \Delta C t}$ formula where $\Delta \Delta C_{\mathrm{t}}=\Delta C_{\mathrm{tTumoural}}-\Delta C_{\mathrm{tNonTumoural}}$.

\section{Statistical analysis}

The data distribution of expression levels being non-Gaussian, non-parametric tests were used for data analyses. Increase in transcript levels of each Tp73 isoform was analysed by signed tests, whereas differences between tumour tissues were tested by the Wilcoxon rank sum test. Each isoform was dichotomised according to its median value. Survival was estimated according to the Kaplan-Meier method and compared between groups by means of the log-rank test. For correlations among the various isoforms and patient age, the threshold cycle differences were used and the Spearman correlation test was applied. Multivariate analyses were performed with the Cox proportional hazard model. Models were fitted for the threshold cycle differences and for the dichotomised variable. All tests were two-sided and the type I error was set at $5 \%$. Statistical analyses were performed with use of SAS Statistical Software (Release 8.02).

\section{RESULTS}

Global analysis of P1 - (P1-p73) and P2 - ( $\Delta$ Np73) generated transcript expression levels conducted in 11 patients shows that P1-p73 transcript levels are increased in low-grade gliomas $\left(P<10^{-3}\right)$ when compared to epileptic tissue samples taken as non-tumoural controls (Table 1C). In contrast, tumour samples displayed no increase of $\Delta \mathrm{Np} 73$ transcript levels. The same observation was carried out in 28 high-grade gliomas $\left(P<10^{-3}\right)$. Because no significant difference was found in P1-p73 transcript expression between high- and low-grade tumours, deregulation of the $\mathrm{P} 1$ promoter that generates the TAp73 and $\Delta \mathrm{TAp} 73$ isoforms was likely to occur early in the tumorigenic process. This upregulation of the P1-p73 transcripts was observed in the centre of the tumours whatever their grade. In contrast, although the high-grade tumour periphery still displayed high levels of the transcript, this expression was significantly $(P=0.031)$ lower on the periphery of low-grade tumours (Table 2).

On the whole, these data suggest that low- and high-grade tumours can be distinguished on the basis of their P1-p73 transcript expression status in their central and peripheral areas. In addition this selective expression of $\mathrm{P} 1-\mathrm{p} 73$ between these areas in low-grade tumours argues in favour of an evolving process.

Analysis of P1-p73 transcripts (TAp73, $\Delta \mathrm{Ex} 2 \mathrm{p} 73$ and $\Delta \mathrm{Ex} 2-$ 3 p73 and $\Delta N^{\prime}$ p73) shows that TAp73, $\Delta \mathrm{Ex} 2 \mathrm{p} 73$ and $\Delta \mathrm{Ex} 2-3 \mathrm{p} 73$ transcripts levels were increased $(P<0.05)$ as compared to the expression spectrum displayed by non-tumoural, control tissue (Table 1C and Figure 2). On the contrary, expression of the $\Delta \mathrm{N}^{\prime}$ isoform in tumour samples did not appear to differ from that of controls. In addition, expressions of these different isoforms were correlated to each other as significant associations were observed between TAp73 and $\Delta$ Ex2p73 levels $\left(P<10^{-2}\right)$ and TAp73 and $\Delta \mathrm{Ex} 2-3 \mathrm{p} 73$ levels $\left(P<10^{-3}\right)$. These data led to the conclusion that, with the exception of the $\Delta N^{\prime}$ isoform, all P1 transcripts were upregulated in the tumours. Again, these findings argued in favour of the early deregulation of the P1 promoter in tumours.

Differences in the expression levels were observed within tumoural and non-tumoural tissue samples. This observation was particularly noticeable for TAp73 and $\Delta \mathrm{Ex} 2-3 \mathrm{p} 73$ transcript levels with a variation of $>30000$-fold and $>10000$-fold within this groups (lowest versus highest expression). A similar observation was reported by Concin et al, 2004 concerning ovarian carcinomas where $\Delta \mathrm{Ex} 2-3 \mathrm{p} 73$ show in normal tissues a variation of $>7000$-fold from the lowest to highest measured. One possible explanation for this variation range is that this variation may reflects perceptibly the heterogeneity of the tumours, patient-topatient variability or both.

Table 2 Normalised expression levels of PI $-\mathrm{p} 73$ and $\Delta \mathrm{Np} 73$ on the periphery of the tumour and at the centre of the tumour in low- and high-grade gliomas

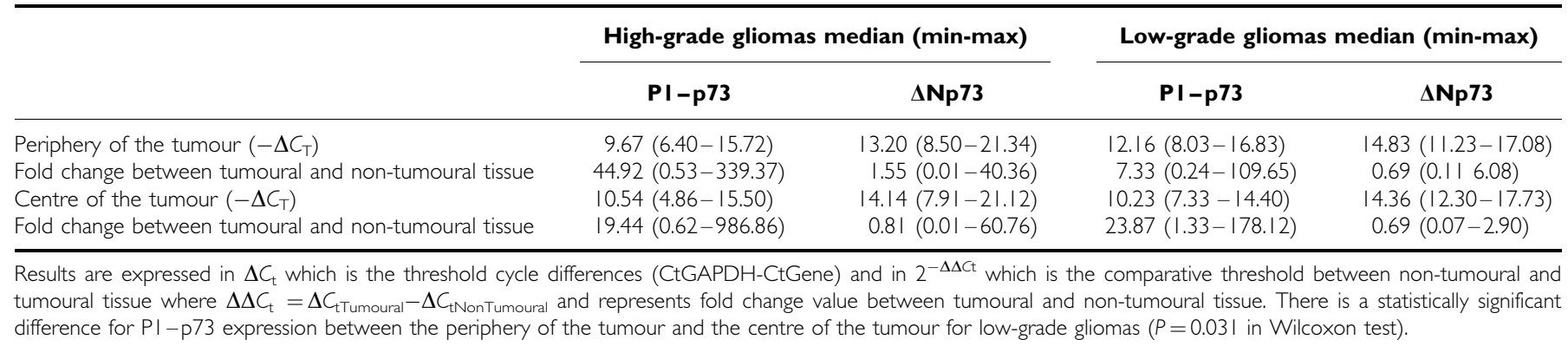



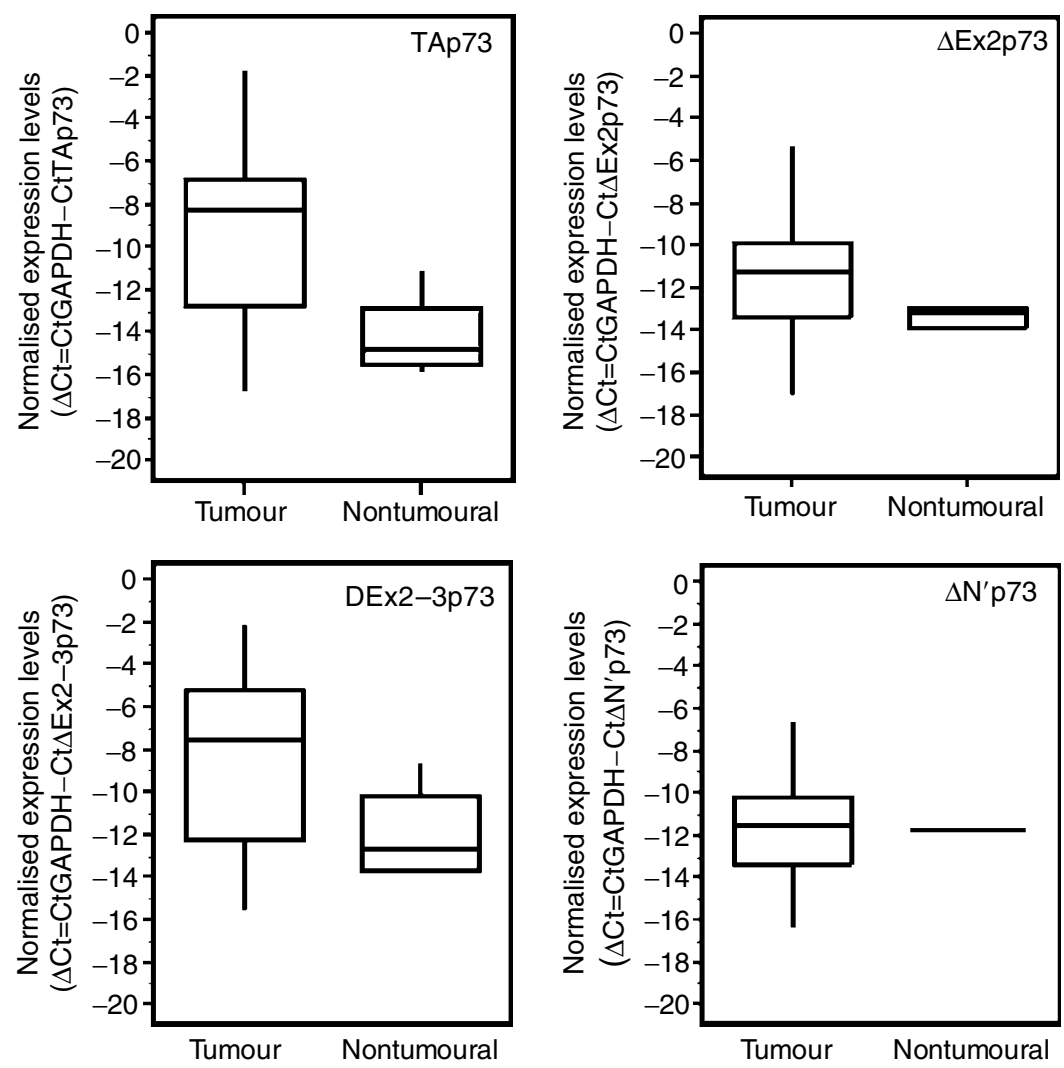

Figure 2 Box plot diagrams showing the normalised expression levels $\Delta C_{t}$ (CtGAPDH-gene) of promoter I- generated Tp73 isoforms (TAp73, $\Delta \mathrm{E} \times 2 \mathrm{p} 73, \Delta \mathrm{E} \times 2-3 \mathrm{p} 73$ and $\Delta \mathrm{N}^{\prime} \mathrm{p} 73$ ) in 23 low-grade gliomas tumours and three non-tumoural tissues. The line within the boxes indicates the median expression level. The top edge of the boxes represent the 75th percentile, the bottom edge, the 25th percentile. The range is shown as a vertical edge.

In order to determine the clinical relevance of these findings with regards to low-grade glioma outcome, survival analysis was performed as regards overall survival rates. Taking as references the median values of mRNA levels of different transcripts - above and below the 50th percentile of the range of the latter - two groups were defined. This approach was dictated by the impossibility of estimating the absolute copy numbers of transcripts. Group 1 included patients in whom the expression levels for each transcript species were higher than their respective median values. Group 2 included patients whose expression levels were lower or equal to the median values. Among the isoforms, expression levels of the $\Delta \mathrm{Ex} 2-3 \mathrm{p} 73$ transcript proved to be a strong prognostic marker for overall survival in our low-grade glioma cohort $(P=0.007$, Figure $3 \mathrm{~A})$. Although 10 out of $12(83 \%)$ $\Delta \mathrm{Ex} 2-3 \mathrm{p} 73$ low expressor patients (group 2) were still alive as of the last follow-up, nine out of eleven ( $82 \%)$ of the high expressor (group 1) patients were deceased. The median overall survival time for high expressor patients was 13 months (IC95\% 4-20) whereas median survival time for low expressor patients was not reached. The high expresssor patients (group 1) had a relative amount of $\Delta$ Ex2-3p73 mRNA > 22-fold compared with non-tumoural epileptic tissue whereas patients from group 2 had a relative amount of $\Delta \mathrm{Ex} 2-3 \mathrm{p} 73 \mathrm{mRNA}<=22$-fold as compared to non-tumoural epileptic tissue.

In multivariate analysis including age and $\Delta \mathrm{Ex} 2-3 \mathrm{p} 7$ expression, age is a significant prognostic factor $(P=0.017)$ and a trend is observed for $\Delta \mathrm{Ex} 2-3 \mathrm{p} 73(P=0.070)$. However, when $\Delta \mathrm{Ex} 2-3$ expression is classified in low and high groups, the only factor that remains significant, even when age is taken into account, is $\Delta \mathrm{Ex} 2-$ 3 p73 $(P=0.025)$.

A similar observation was also carried out, but to a lesser extent, for $\Delta \mathrm{Ex} 2 \mathrm{p} 73$ as high expressor patients had a worse outcome than did low expressor patients ( $P=0.043$ and Figure 3B). In contrast, TAp73 and $\Delta \mathrm{N}^{\prime}$ p73 transcripts did not appear to be involved as the survival rates in the two groups were similar $(P=0.144$ and $P=0.906$, respectively Figure $3 \mathrm{C}$ and $\mathrm{D}$ ).

As we know that the neutralising role of these transdominant forms take place through oligomerisation with TAp73 and p53 proteins it is crucial to confirm that RNA overexpression of the pronostic splice variants as far as proteins are concerned. Unfortunately no specific antibodies for any of the $\Delta \mathrm{TAp} 73 \mathrm{forms}$ currently exist. We cannot therefore propose the existence of a dominant negative mechanism with $\Delta \mathrm{Ex} 2-3 \mathrm{p} 73$ and $\Delta \mathrm{Ex} 2 \mathrm{p} 73$ variants in low-grade gliomas. However, as mRNA expression of $\Delta \mathrm{Ex} 2-3 \mathrm{p} 73$ and to a lesser extent $\Delta \mathrm{Ex} 2 \mathrm{p} 73$ appeared to be correlated to low-grade gliomas patients survival and to be a strong prognostic marker in these tumours, our data, although suggestive on the nefastious role of these transcripts, do not allow to definitely conclude on this point.

\section{DISCUSSION}

\section{Tp73 involvement in glial tumorigenesis}

The role of the Tp73 gene in oncogenic process has been underlined by several reports dealing with different tumour types. Results including ours have clearly shown that the expression of the gene rises frequently in a number of tumours although considerable variations in the levels of transcripts from patient to patient can be noted (Concin et al, 2004). A previous work dealing with gliomas and using a semiquantitative RT-PCR assay has shown that a similar situation also exists in this type of tumour as overexpression of TAp73 mRNA occurred in high-grade gliomas 

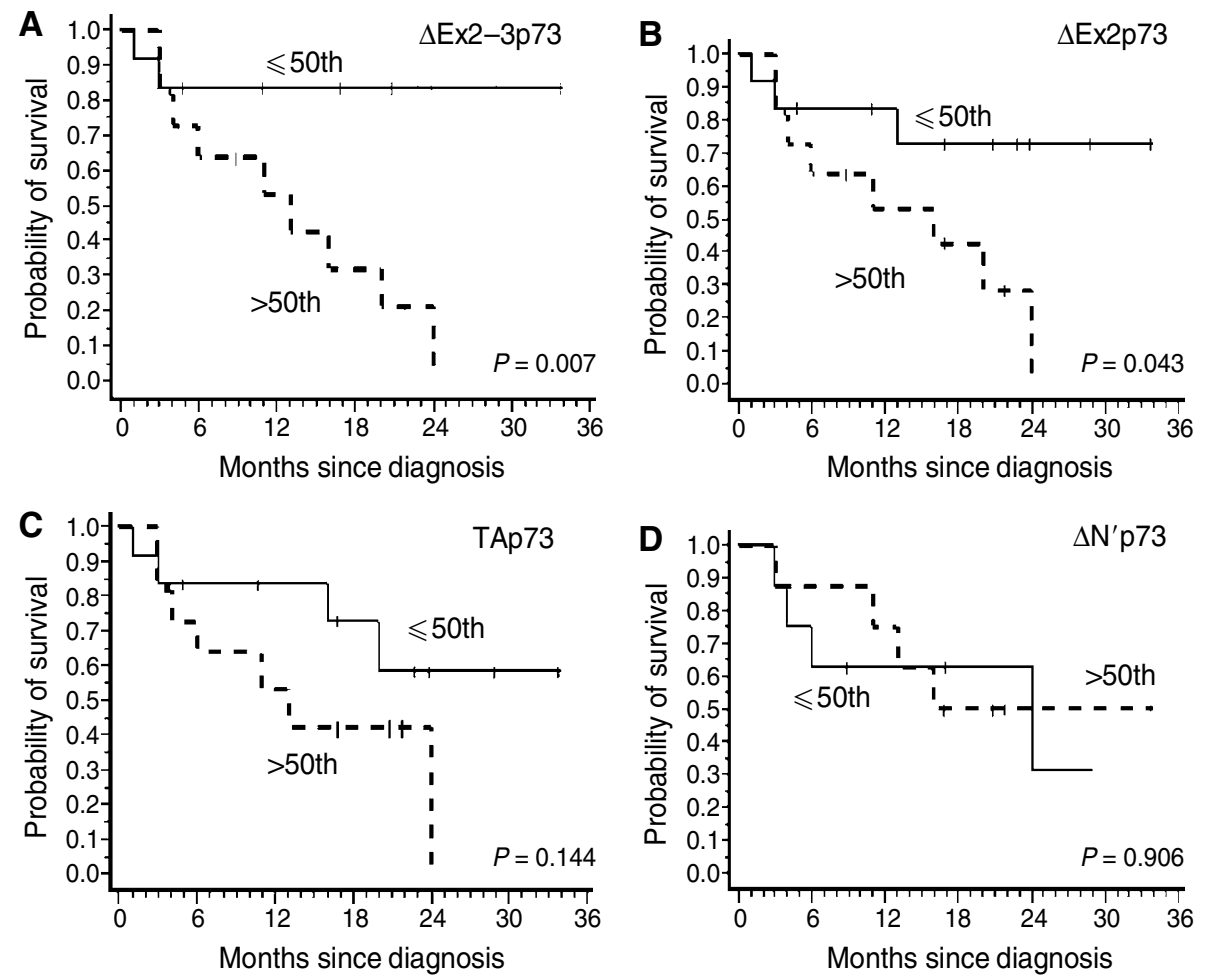

Figure 3 Kaplan-Meier survival curves in 23 low-grade gliomas. Two groups were defined taking as reference the median values of the mRNA levels of different transcripts (above and below the 50th percentile of the range of the different transcripts). (A) $\Delta$ Ex 2-3p73, (B) $\Delta$ Ex2p73, (C) TAp3 and (D) $\Delta \mathrm{N}^{\prime} \mathrm{p} 73$.

whereas only a few tumours displayed $\Delta \mathrm{Np} 73$ expression (Ugur et al, 2004). The present study confirms these data and extends them by reporting increase in transcript levels of the P1-generated Tp73 transcripts, namely TAp73, $\Delta \mathrm{Ex} 2 \mathrm{p} 73$ and $\Delta \mathrm{Ex} 2-3 \mathrm{p} 73$, (but not $\left.\Delta \mathrm{N}^{\prime} \mathrm{p} 73\right)$ in a cohort of low-grade adult gliomas. Interestingly $\Delta$ Np73 transcript does not appear to differ from that of control samples. Thus, deregulation of the P1 promoter and emergence of particular RNA populations are likely to be an early event as this deregulation is likewise observed in high-grade gliomas and could possibly influence cell evolution toward malignancy.

The abundance of Tp73 mRNA isoforms in specific cell type is complex and is likely to result from differential expression, RNA stability and splicing. In our study, increase in transcript levels of P1-generated isoforms could be explained by coupled mechanisms: activation of the $\mathrm{P} 1$ promoter itself and selective splicing process leading to an increase of certain mRNA species (TAp73, $\Delta$ Ex2p73 and $\Delta \mathrm{Ex} 2-3 \mathrm{p} 73$ but not $\left.\Delta \mathrm{N}^{\prime} \mathrm{p} 73\right)$. The expression of some transcripts could be restricted to particular areas of the tumour suggesting differential splicing in this area. Addressing this question would require to perform RNA status analysis of at the individual tumour cells level.

The selective patterns of expression of the transdominantTp73 gene isoforms observed in a variety of tumours ( $\mathrm{Ng}$ et al, 2000; Cui et al, 2005), should reflect a particular distribution of the factors that contribute to splicing such as HnRNP proteins. Incidentally, several reports have shown the existence of particular populations of HnRNP proteins in a variety of tumours suggesting that their expression is implicated in selective activation of gene isoforms expression (Carpenter et al, 2006).

Tumour-specific variants have been reported to affect transcript stability and thus, accumulation of the variant transcripts could result from an increased stability. Regarding this point, impaired mRNA turnover and stability are shown to play a critical role in the activation of specific genes during the cellular response to mitogens, immunological triggers stressful stimuli and differentiation agents (Bashirullah et al, 2001; Fan et al, 2002). Several parameters control the mRNA turnover: RNA-binding proteins (RBPs) that bind to specific RNA sequences and either increase or decrease the transcript half-life (Brennan and Steitz, 2001; Gorospe, 2003;); regions located in the $3^{\prime}$-untranslated regions (3'-UTRs) of mRNA. In the latter, differences in $3^{\prime}$-UTRs of splice variants could be recognised by proteins expressed specifically in some normal tissues or tumoural tissues or in a particular areas of the tumour (Jensen and Whitehead, 2004). Finally, the RBP HuR, upon binding to RNA HuR, has been shown to stabilise it and alter its translation (Brennan and Steitz, 2001; Gorospe, 2003; Mazan-Mamczarz et al, 2003).

Another aspect is the differential expression of Tp73 transcripts in the peripheral and central areas of low-grade tumours as compared to high-grade gliomas that overexpress the gene in both areas. Beside the possibility of discriminating between high- and low-grade tumours, this result suggests that malignant cells from the periphery of low-grade gliomas differ from those located in the tumour centre. By using the two dimensional profiling strategy Li et al (2006) found a large number of genes that showed coordinated changes in transcript abundance and splicing, indicating that many distinct steps in gene expression from transcription, stability control and splicing may be coupled in different cell types (Li et al, 2006). In view of this observation it is tempting to speculate that the peripheral cells in low-grade tumours that differ from their central area congeners may evolve toward a more malignant stage or disappear to be replaced by tumour cells coming from the centre. We are considering the possibility of addressing these hypothesis by carrying out transcriptome analysis of tumour areas isolated by microdissection. 


\section{Tp73 isoform prognostic value}

A survival analysis aimed at determining the clinical relevance of the increase in $\triangle \mathrm{TAp} 73$ isoform transcript levels in low-grade adult gliomas showed that patients with higher $\Delta \mathrm{Ex} 2-3 \mathrm{p} 73$ contents in their tumours presented shorter survival than those with lower amounts of this transcript. Strikingly, increase in the TAp73 isoform transcript levels was also found in all patients, thereby arguing in favour of a dominant negative role for the $\Delta \mathrm{Ex} 2-3 \mathrm{p} 73$ isoform in survival. This situation mimics that of ovarian tumours where a better overall survival was noted for patients exhibiting low expression of the $\Delta \mathrm{N}^{\prime} \mathrm{p} 73$ isoform than for those with high expression, whatever the levels of TAp73 in the tumours (Concin et al, 2004). Moreover, $\Delta \mathrm{Ex} 2-3 \mathrm{p} 73$ is a prognostic factor in low-grade gliomas even when age is considered.

Up until now, the status of the Tp73 gene involving its various isoforms has been examined in ovarian tumours, hepatocarcinomas, vulval cancers, oesophagus and gliomas (this report) $(\mathrm{Ng}$ et al, 2000; Cui et al, 2005). Although these studies point to increase in transcript levels of transdominant $\Delta \mathrm{TAp} 73$ isoforms, it may be noted that, depending on the tumour pathology, a selective expression of one of the transdominant isoforms appears to be detected in certain tumour types, supporting the notion that among the various P1-generated isoforms, differential regulation exists. Given the necessarily limited number of patients included in this study, our data strongly suggest that the $\Delta \mathrm{Ex} 2-3 \mathrm{p} 73$ isoform is selectively expressed in gliomas. It will be of interest to extend these observations to other tumour types in order to determine whether a more specific expression spectrum exists. Obviously, such selective patterns should reflect an abnormal distribution in tumour cells of the factors that contribute to splicing. In any case, whatever the identity of the expressed transdominant $\Delta \mathrm{TAp} 73$ isoform, the biological significance directly relies on the neutralising role of this transdominant form to TAp73 and/or p53.
Although the gross natural history of adult low-grade glioma is known, a given individual outcome remains unpredictable. This lack of individual predictive factors results in daunting difficulties once therapeutic decisions are involved. When a low-grade glioma is newly diagnosed, clinicians have to decide when to proceed to treatment: not too early because therapeutic means - extensive surgical removal, radiation therapy, chemotherapy - are contestable as pertains to a benign tumour, but not too late either, that is before progression to higher grades of these infiltrating tumours. Up until now the indicators of such a change are clinical symptoms and imaging changes (e.g. contrast enhancement) but they only accompany, and fail to anticipate clinical tumour progression. New criteria allowing for earlier detection of tumour grade progression and correspondingly more expeditious therapeutic adaptation would be of heightened value as regards optimal treatment of these patients. Nevertheless, the use of $\Delta \mathrm{Ex} 2-3 \mathrm{p} 73$ as such an indicator has one limitation, which is inherent to our methodology: as there exists no absolute quantification of this increase in transcript levels, it has got to be compared with a non-tumoural control group.

In conclusion, to the best of our knowledge this is the first report of a single molecular prognostic marker criterion for lowgrade glioma survival in adults. This could lead to early treatment of the newly diagnosed adult patients harbouring lowgrade gliomas and exhibiting increase in transcript levels of $\Delta \mathrm{Ex}$ $2-3 \mathrm{p} 73$.

\section{ACKNOWLEDGEMENTS}

This work was supported by Grants from PHRC (Projet Hospitalier de Recherche Clinique) 2003, and Ligue contre le Cancer: Comité Départemental de la Vienne.

\section{REFERENCES}

Applied Biosystems User Bulletin no. (1997) (P/N 4303559). Relative quantitation of gene expression

Bashirullah A, Cooperstock RL, Lipshitz HD (2001) Spatial and temporal control of RNA stability. Proc Natl Acad Sci USA 98(13): 7025-7028

Bergamaschi D, Gasco M, Hiller L, Sullivan A, Syed N, Trigiante G, Yulug I, Merlano $M$, Numico $G$, Comino A, Attard $M$, Reelfs $O$, Gusterson B, Bell AK, Heath V, Tavassoli M, Farrell PJ, Smith P, Lu X, Crook T (2003) p53 polymorphism influences response in cancer chemotherapy via modulation of p73-dependent apoptosis. Cancer Cell 3(4): $387-402$

Brennan CM, Steitz JA (2001) HuR and mRNA stability. Cell Mol Life Sci 58(2): 266-277. Review

Carpenter B, Mackay C, Alnabulsi A, Mackay M, Telfer C, Melvin WT, Murray GI (2006) The roles of heterogeneous nuclear ribonucleoproteins in tumour development and progression. Biochim Biophys Acta 1765(2): $85-100$

Casciano I, Mazzocco K, Boni L, Pagnan G, Banelli B, Allemanni G, Ponzoni M, Tonini GP, Romani M (2002) Expression of DeltaNp73 is a molecular marker for adverse outcome in neuroblastoma patients. Cell Death Differ 9(3): $246-251$

Concin N, Becker K, Slade N, Erster S, Mueller-Holzner E, Ulmer H, Daxenbichler G, Zeimet A, Zeillinger R, Marth C, Moll UM (2004) Transdominant $\Delta$ Tap73 isoformsare frequently up-regulated in ovarian cancer. Evidence for their role as epigenetic p53 inhibitors in vivo. Cancer Res 64: 2449-2460

Cui R, He J, Mei R, deFromentel CC, Martel-Planche G, Taniere P, Hainaut P (2005) Expression of p53, p63, and p73 isoforms in squamous cell carcinoma and adenocarcinoma of esophagus. Biochem Biophys Res Commun 25, in press

Fan J, Yang X, Wang W, Wood III WH, Becker KG, Gorospe M (2002) Global analysis of stress-regulated mRNA turnover by using cDNA arrays. Proc Natl Acad Sci USA 6;99(16): 10611-10616
Flores ER, Tsai KY, Crowley D, Sengupta S, Yang A, McKeon F, Jacks T (2002) p63 and p73 are required for p53-dependent apoptosis in response to DNA damage. Nature 4;416(6880): $560-564$

Gorospe M (2003) HuR in the mammalian genotoxic response: posttranscriptional multitasking. Cell Cycle 2(5): 412-414. Review

Grob TJ, Novak U, Maisse C, Barcaroli D, Luthi AU, Pirnia F, Hugli B, Graber HU, De laurenzi V, Fey MF, Melino G, Tobler A (2001) Human delta Np73 regulates a dominant negative feedback loop for TAp73 and p53. Cell Death Differ 8(12): 1213-1223

Irwin MS, Kondo K, Marin MC, Cheng LS, Hahn WC, Kaelin Jr WR (2003) Chemosensitivity linked to p73 function. Cancer Cell 3(4): $403-410$

Jensen LE, Whitehead AS (2004) The $3^{\prime}$ untranslated region of the membrane-bound IL-1R accessory protein mRNA confers tissue-specific destabilization. J Immunol 173(10): 6248-6258

Kleihues P, Cavenee WK (2000) Tumours of the nervous system: pathology and genetics World Health Organization Classification of Tumours. IARC Press: Lyon

Li HR, Wang-Rodriguez J, Nair TM, Yeakley JM, Kwon YS, Bibikova M, Zheng C, Zhou L, Zhang K, Downs T, Fu XD, Fan JB (2006) Twodimensional transcriptome profiling: identification of messenger RNA isoform signatures in prostate cancer from archived paraffin-embedded cancer specimens. Cancer Res 66(8): 4079-4088

Livak KJ, Schmittgen TD (2001) Analysis of relative gene expression data using real-time quantitative PCR and the 2(-Delta Delta C(T)) method. Methods 25(4): $402-408$

Mazan-Mamczarz K, Galban S, Lopez de Silanes I, Martindale JL, Atasoy U, Keene JD, Gorospe M (2003) RNA-binding protein HuR enhances p53 translation in response to ultraviolet light irradiation. Proc Natl Acad Sci USA 100(14): $8354-8359$

Melino G, De Laurenzi V, Vousden KH (2002) p73: Friend or foe in tumorigenesis. Nat Rev Cancer 2(8): 605-615. 25 
Tp73 $\Delta$ Ex2-3 isoforms in low-grade glioma patients

$M$ Wager et al

Nakagawa T, Takahashi M, Ozaki T, Watanabe Ki K, Todo S, Mizuguchi H, Hayakawa T, Nakagawara A (2002) Autoinhibitory regulation of p73 by Delta Np73 to modulate cell survival and death through a p73-specific target element within the Delta Np73 promoter. Mol Cell Biol 22(8): 2575-2585

Ng SW, Yiu GK, Liu Y, Huang LW, Palnati M, Jun SH, Berkowitz RS, Mok SC (2000) Analysis of p73 in human borderline and invasive ovarian tumor. Oncogene 6;19(15): 1885-1890

Petrenko O, Zaika A, Moll UM (2003) deltaNp73 facilitates cell immortalization and cooperates with oncogenic Ras in cellular transformation in vivo. Mol Cell Biol 23(16): 5540-5555
Stiewe T, Theseling CC, Putzer BM (2002a) Transactivation-deficient Delta TA-p73 inhibits p53 by direct competition for DNA binding: implications for tumorigenesis. J Biol Chem 19;277(16): 14177-14185

Stiewe T, Zimmermann S, Frilling A, Esche H, Putzer BM (2002b) Transactivation-deficient DeltaTA-p73 acts as an oncogene. Cancer Res 62(13): $3598-3602$

Ugur H, Sayan AE, Ozdamar SO, Kanpolat Y, Ozturk M (2004) Expression of TAP73 and DeltaNP73 in malignant gliomas. Oncol Rep 11(6): $1337-1341$ 